\title{
ON FINITE ANTI-PLANE SHEAR FOR IMCOMPRESSIBLE ELASTIC MATERIALS
}

\author{
JAMES K. KNOWLES
}

(Received 27 August 1976)

\begin{abstract}
This paper is concerned with deformations corresponding to antiplane shear in finite elastostatics. The principal result is a necessary and sufficient condition for a homogeneous, isotropic, incompressible material to admit nontrivial states of anti-plane shear. The condition is given in terms of the strain energy density characteristic of the material and is tllustrated by means of special examples.
\end{abstract}

\section{Introduction}

A solid body which occupies a cylindrical region in its undeformed configuration is said to be deformed to a state of anti-plane shear if the displacement of each particle is parallel to the generators of the cylinder and independent of the axial position of the particle. Deformations of this kind are receiving increasing attention, especially in connection with problems which involve cracks or notches and are therefore of interest in the study of fracture'. Although of less direct practical interest than their counterparts in plane strain or plane stress, such problems acquire a measure of importance from their comparative analytical simplicity. They are thus well suited to the role of pilot problems to be studied for the purpose of uncovering qualitative features - the effect of nonlinearity on crack-tip singularities, for example which present much more formidable technical obstacles in plane strain or generalized plane stress ${ }^{2}$.

1. A sample of the relatively recent literature pertanning to static or dynamic anti-plane shear of bodies containing cracks is furnished by references [1], [2], [4], [5], [9], [10], [13] and [14].

2 . In the setting of finte elasticity, the comparative simplicity of crack problems in anti-plane shear - as opposed, say, to tension-crack problems - is aptly illustrated by the contrast between the analyses to be found in [10] on the one hand and [11], [12] on the other. 
Most of the analytical effort alluded to above in connection with anti-plane shear is directed toward problems which are formulated either in terms of the classical linearized theory of elasticity or on the basis of theories in which displacement gradients are assumed infinitesimally small, but a nonlinear relation between stress and strain is used in place of the conventional linear one. Exceptions, however, may be found in the work reported in [10] and [13], where the governing equations are those of the fully nonlinear equilibrium theory of finite elasticity.

The first detailed study of anti-plane shear in the context of finite elasticity appears to have been that of Adkins [3], who considered incompressible isotropic bodies with a view toward applications to rubber-like materials'. It is implicit in Adkins' discussion that the two final differential equations to which he reduced the theory restrict in general not only the form of the outof-plane displacement in the problem at hand, but also the strain energy density characteristic of the material under consideration. Thus, although any elastic material - even a compressible one - admits deformations corresponding to simple shear ${ }^{2}$, it is not to be expected that an arbitrary isotropic incompressible material will sustain nontrivial states of anti-plane shear in the absence of body forces ${ }^{3}$. For the special case of a material of the so-called Mooney type, no inconsistency arises between the two differential equations mentioned above, and nontrivial states of anti-plane shear are possible in this instance. Much of the detailed analysis in [3] applies only to this special case, which, to be sure, is of great importance for the description of the mechanical behavior of rubber.

It is known from the results in [10] and [13] that there are strain energy densities other than that of the Mooney type which admit nontrivial states of anti-plane shear. The question of determining necessary and sufficient conditions on the strain energy density in order that the corresponding incompressible material shall admit nontrivial states of anti-plane shear was not considered in [3], [10] or [13] and appears to be still open. This issue is the subject of the present paper.

In the following section the theory of finite anti-plane shear following a homgeneous axial pre-stretch is sketched for isotropic, incompressible elastic bodies. While the content of Section 2 is by no means new, the presentation differs somewhat from those given in [3] and [8]. In Section 3 the central question is formulated, and the desired necessary and sufficient condition on

1. A summary of Adkins' results may be found in Chapter II of [8].

2. In simple shear the displacement gradient is constant.

3. An entirely analogous issue arises for compressible elastic materials. 
the strain energy density is derived for anti-plane shear deformations which are unaccompanied by an axial pressure gradient. The principal tool used in the derivation is a transformation of hodograph type. Some of the consequences of the restriction derived in Section 3 are discussed in the final section.

\section{Finite anti-plane shear}

Consider a body of imcompressible, isotropic elastic material which in its undeformed state occupies a region $\mathscr{R}$. A deformation of the body is a smooth, one-to-one relationship of the form

$$
y=y(x), \quad x \in \mathscr{R},
$$

between the position vectors $x$ and $y$ locating a given particle in the undeformed and deformed configurations, respectively. The deformation gradient tensor $\boldsymbol{F}$ is defined by

$$
\boldsymbol{F}=\nabla \boldsymbol{y}
$$

and the incompressibility of the material requires that

$$
\operatorname{det} \boldsymbol{F} \equiv 1
$$

for every admissible deformation. The left Cauchy-Green tensor associated with $(2.1)$ is

$$
\boldsymbol{G}=\boldsymbol{F F}^{\boldsymbol{T}},
$$

where $\boldsymbol{F}^{T}$ stands for the transpose of $\boldsymbol{F}$. The first two fundamental invariants of $G$ are

$$
I_{1}=\operatorname{Tr} \boldsymbol{G}, \quad I_{2}=\frac{1}{2}\left[(\operatorname{Tr} \boldsymbol{G})^{2}-\operatorname{Tr}(\boldsymbol{G})^{2}\right] .
$$

The mechanical response of a material of the type under consideration is determined by the strain energy $W$ per unit undeformed volume; $W$ depends only on the invariants $I_{1}$ and $I_{2}: W=W\left(I_{1}, I_{2}\right)$. The stress-deformation relation may be taken in the form ${ }^{2}$

$$
\boldsymbol{\sigma}=2 \frac{\partial W}{\partial I_{1}} \boldsymbol{F}+2 \frac{\partial W}{\partial I_{2}}\left(I_{1} \mathbf{1}-\boldsymbol{G}\right) \boldsymbol{F}-p\left(\boldsymbol{F}^{T}\right)^{-1},
$$

where $\sigma$ is the nominal (or Piola) stress tensor (force per unit undeformed area), $p$ is an arbitrary hydrostatic pressure, 1 stands for the unit tensor, and

1. The third invariant $I_{3}=\operatorname{det} \boldsymbol{G}=\operatorname{det} \boldsymbol{F}^{2}$ has the value unity because of (2.3). It is possible to show that, if $\operatorname{det} G=1$, then always $I_{1} \geqq 3, I_{2} \geqq 3$.

2. See Equations (43A.3), (47.8) and (86.9) of [15]. 
$\left(\boldsymbol{F}^{T}\right)^{-1}$ indicates the inverse of $\boldsymbol{F}^{T}$. The true (or Cauchy) stress tensor $\tau$ is related to $\boldsymbol{\sigma}$ according to

$$
\tau=\boldsymbol{\sigma} \boldsymbol{F}^{r}
$$

in the case of incompressible materials; $\tau$ is symmetric, but $\sigma$ in general is not.

In the absence of body forces, the local equilibrium condition for the body may be written as

$$
\operatorname{div} \boldsymbol{\sigma}=\mathbf{0} \text { on } \mathscr{R} \text {. }
$$

For the study of anti-plane shear, one considers the case of a cylindrical region $\mathscr{R}$, chooses a system of cartesian coordiantes $x_{1}, x_{2}, x_{3}$ with the $x_{3}$-axis parallel to the generators of $\mathscr{R}$, and examines deformations (2.1) of the special form

$$
y_{1}=\lambda^{-\frac{1}{2}} x_{1}, \quad y_{2}=\lambda^{-\frac{1}{2}} x_{2}, \quad y_{3}=\lambda x_{3}+u\left(x_{1}, x_{2}\right) .
$$

Here $y_{1}$ and $x_{1}$ are the components in the chosen coordinate frame of $\boldsymbol{y}$ and $\boldsymbol{x}$, respectively, $\lambda$ is a positive constant, and $u$ is a function which is twice continuously differentiable on the open cross-section $\mathscr{D}$ of the cylinder. The deformation (2.9) may be thought of as one in which the body first undergoes an axial elongation (or contraction) of stretch ratio $\lambda$ (regarded as given), and is then subjected to an anti-plane shear with out-of-plane displacement $u$. The matrices of components (in the given coordinate frame) of the tensors $\boldsymbol{F}$ and $\boldsymbol{G}$ of (2.4) may be readily calculated for deformations of the form (2.9):

$$
\begin{gathered}
\boldsymbol{F}=\left(\begin{array}{lll}
\lambda^{-\frac{1}{2}} & 0 & 0 \\
0 & \lambda^{-\frac{1}{2}} & 0 \\
u, 1 & u, 2 & \lambda
\end{array}\right), \\
\boldsymbol{G}=\left(\begin{array}{lll}
\lambda^{-1} & 0 & \lambda^{-\frac{1}{2}} u, 1 \\
0 & \lambda^{-1} & \lambda^{-\frac{1}{2}} u, 2 \\
\lambda^{-\frac{1}{2}} u, 1 & \lambda^{-\frac{1}{2}} u, \lambda^{2}+|\nabla u|^{2}
\end{array}\right) ;
\end{gathered}
$$

here

$$
|\nabla u|^{2}=u,{ }_{\alpha} u,{ }_{\alpha}
$$

1. Latin subscripts take the values $1,2,3$, while Greek subscripts are limited to the range 1, 2. Repeated subscripts are summed over the appropriate range. A subscript preceded by a comma indicates differentiation with respect to the corresponding $x$-coordinate.

2. The same symbol is used for a tensor (e.g. F) and its matrix of components. 
One notes from (2.10) that the incompressibility condition (2.3) is automatically fulfilled for every $\lambda$ and $u$. The invariants of (2.5) may be determined from (2.11) to be

$$
I_{1}=\lambda^{2}+2 \lambda^{-1}+|\nabla u|^{2}, \quad I_{2}=\lambda^{-2}+2 \lambda+\lambda^{-1}|\nabla u|^{2} .
$$

When $\lambda=1$, so that there is no pre-stretch, (2.13) reduce to

$$
I_{1}=I_{2}=3+|\nabla u|^{2} \quad(\lambda=1) .
$$

If the values of $F, G, I_{1}$ and $I_{2}$ are substituted from (2.10)-(2.13) into (2.6), the components of nominal stress which follow are

$$
\begin{gathered}
\sigma_{\alpha \beta}=\lambda^{-\frac{1}{2}}\left[2 \frac{\partial W}{\partial I_{1}}+2\left(\lambda^{2}+\lambda^{-1}+|\nabla u|^{2}\right) \frac{\partial W}{\partial I_{2}}-\lambda p\right] \delta_{\alpha \beta}-2 \lambda^{-\frac{1}{2}} \frac{\partial W}{\partial I_{2}} u_{, \alpha} u, \beta \\
\sigma_{\alpha 3}=\left(-2 \lambda^{\frac{1}{2}} \frac{\partial W}{\partial I_{2}}+\lambda^{-\frac{1}{2}} p\right) u,_{\alpha} \\
\sigma_{3 \alpha}=2\left(\frac{\partial W}{\partial I_{1}}+\lambda^{-1} \frac{\partial W}{\partial I_{2}}\right) u,_{\alpha} \\
\sigma_{33}=2 \lambda \frac{\partial W}{\partial I_{1}}+4 \frac{\partial W}{\partial I_{2}}-\lambda^{-1} p
\end{gathered}
$$

When the above components of $\sigma$ are in turn substituted into the component form of the equilibrium conditions (2.8), the latter yield

$$
\begin{gathered}
q_{, \alpha}+\left(2 \frac{\partial W}{\partial I_{1}} u_{\alpha} u_{, \beta}\right)_{, \beta}-p_{, 3} u_{, \alpha}=0, \\
{\left[2\left(\frac{\partial W}{\partial I_{1}}+\lambda^{-1} \frac{\partial W}{\partial I_{2}}\right) u_{, \beta}\right]_{, \beta}-\lambda^{-1} p,_{3}=0,}
\end{gathered}
$$

where the abbreviation

$$
q=\lambda p-2 \frac{\partial W}{\partial I_{1}}-2\left(\lambda^{2}+\lambda^{-1}+|\nabla u|^{2}\right) \frac{\partial W}{\partial I_{2}}
$$

has been introduced. The fact that $u_{\alpha}$, and therefore $I_{1}, I_{2}$, are independent of $x_{3}$ permits one to infer from (2.20) that $p, 3$ has this same property. Thus $p$, and therefore $q$, are linear in $x_{3}$. It then follows from (2.19), (2.21) that $q, \alpha$ and $p, \alpha$ are independent of $x_{3}$. Thus

$$
p\left(x_{1}, x_{2}, x_{3}\right)=c x_{3}+\bar{p}\left(x_{1}, x_{2}\right)
$$

1. $\delta_{\alpha \beta}$ is the Kronecker delta. 
where $c$ is a constant - to be called here the axial pressure gradient - and $\bar{p}$ is an undetermined function. In view of (2.22), the equilibrium equations (2.19), (2.20) reduce to

$$
\begin{gathered}
(q-c u)_{, \alpha}+\left(2 \frac{\partial W}{\partial I_{2}} u,_{\alpha} u,_{\beta}\right),_{\beta}=0, \\
{\left[2\left(\frac{\partial W}{\partial I_{1}}+\lambda^{-1} \frac{\partial W}{\partial I_{2}}\right) u_{\beta}\right]_{, \beta}-\lambda^{-1} c=0 .}
\end{gathered}
$$

The system (2.23), (2.24) consists of three differential equations for the two functions $q$ and $u$ which must be satisfied (for a suitable value ${ }^{1}$ of $c$ and for the given constant $\lambda$ ) if the deformation (2.9) is to be possible for the material characterized by the given strain energy density $W$.

A further reduction of the first two equilibrium equations (2.23) may be obtained by eliminating $q-c u$ by appropriate cross-differentiation if $q$ and $u$ are twice and three-times continuously differentiable, respectively. The result is

$$
\varepsilon_{\alpha \gamma}\left(2 \frac{\partial W}{\partial I_{2}} u,_{\alpha} u, \beta\right),{ }_{\beta \gamma}=0,
$$

where $\varepsilon_{\alpha \gamma}$ is the two-dimensional alternator: $\varepsilon_{11}=\varepsilon_{22}=0, \varepsilon_{12}=-\varepsilon_{21}=1$. This equation was obtained by Adkins $^{2}$, who preferred to use $y_{\alpha}$ rather than $x_{\alpha}$ as independent variables. The equivalent of all of the preceding analysis, in fact, was given by Adkins and may be found in $\$ 2.20$ of [8], except that the presentation there (as well as in [3]) is "Eulerian", in contrast to the "Lagrangian" approach adopted here.

For a given material - and thus for a given $\mathrm{W}-$ it is to be expected that (2.23), (2.24) (or (2.24), (2.25)) constitute an over-determined system in general, in the sense that only very special solutions $u$ of $(2.24)$ will be consistent with (2.23) (and hence with (2.25)). It was shown by Adkins in [3], however, that if $W$ has the Mooney form

$$
W\left(I_{1}, I_{2}\right)=A\left(I_{1}-3\right)+B\left(I_{2}-3\right),
$$

where $A$ and $B$ are material constants, then every solution $u$ of (2.24) automatically satisfies (2.25). It is obvious this statement is also true for strain energy densities $W$ which are independent of $I_{2}$ :

1. The constant $c$ is normally determined from the boundary conditions in a particular boundary value problem. If the traction acting along a generator of the cylindrical boundary is independent of $x_{3}$ in such a problem, then $c=0$; see, for example, the crack problem treated in [10].

2. See Eq. (2.20.13), p. 85 of [8]. 


$$
W\left(I_{1}, I_{2}\right)=\hat{W}\left(I_{1}\right) \text {. }
$$

A class of boundary value problems for idealized materials of this latter type was examined in [10] as well as in [13]. One can readily confirm that if $W$ is of the form

$$
W\left(I_{1}, I_{2}\right)=\hat{W}\left(I_{1}\right)+\hat{W}\left(I_{2}\right)
$$

then, as Lo [13] has observed in a speciai case, (2.25) and (2.24) are consistent in the absence of axial pre-stretch (i.e. for $\lambda=1$, so that (2.14) holds), in the sense that every solution of (2.24) also satisfies (2.25).

A necessary and sufficient condition on $W$ for the simultaneous satisfaction of (2.23) and (2.24) in the case of zero pressure gradient $(c=0)$ is obtained in the following section.

\section{Nontrivial states of anti-plane shear}

In the present section, the system (2.23), (2.24) is considered for the case in which the axial pressure gradient is absent', so that $c=0$. It is convenient to introduce the functions

$$
\left.\begin{array}{l}
F\left(|\nabla u|^{2} ; \lambda\right)=2\left[\frac{\partial W}{\partial I_{1}}\left(I_{1}, I_{2}\right)+\lambda^{-1} \frac{\partial W}{\partial I_{2}}\left(I_{1}, I_{2}\right)\right], \\
G\left(|\nabla u|^{2} ; \lambda\right)=2 \frac{\partial W}{\partial I_{2}}\left(I_{1}, I_{2}\right),
\end{array}\right\}
$$

where, in accordance with (2.13), $I_{1}, I_{2}$ are given by

$$
I_{1}=\lambda^{2}+2 \lambda^{-1}+|\nabla u|^{2}, \quad I_{2}=\lambda^{-2}+2 \lambda+\lambda^{-1}|\nabla u|^{2} .
$$

In terms of $F, G$, the systems (2.23), (2.24) can be written as follows when $c=0$ :

$$
\begin{gathered}
q_{, \alpha}+\left(G u,_{\alpha} u,_{\beta}\right)_{, \beta}=0 \text { on } \mathscr{D}, \\
\left(F u,_{\beta}\right)_{, \beta}=0 \text { on } \mathscr{D} .
\end{gathered}
$$

The strain energy density $W$ is assumed to be a given, three times continuously differentiable function of $I_{1}, I_{2}$ which is such that the following inequality is satisfied:

$$
\frac{d}{d R}\left[R F\left(R^{2} ; \lambda\right)\right]>0 \text { for all } R \geqq 0, \lambda>0,
$$

where $F$ is defined in (3.1). For a fixed $R \geqq 0$, this inequality assures the ellipticity $^{2}$ of the quasilinear partial differential equation (3.4) at a solution $u$

1. Note from (2.22) that when $c=0, p$ and hence the stresses are independent of $x_{3}$.

2. See pp. $163-164$ of [7]. 
and at a point $\left(x_{1}, x_{2}\right)$ for which $\left|\nabla u\left(x_{1}, x_{2}\right)\right|=R$. Thus (3.5) guarantees that (3.4) is elliptic at every solution $u$ and at every point in $\mathscr{D}$. Moreover, it follows from (3.5) that

$$
F\left(R^{2} ; \lambda\right)>0 \text { for } R \geqq 0, \lambda>0 .
$$

It may be noted that the Mooney material described by the special choice (2.26) of $W$ satisfies (3.5) if and only if the material moduli $A$ and $B$ are positive and nonnegative, respectively.

Regardless of the special form of $W$, the three differential equations (3.3), (3.4) are satisfied by the choice

$$
u=k_{\alpha} x_{\alpha}, \quad q=\text { constant on } \mathscr{D},
$$

where $k_{1}$ and $k_{2}$ are constants. Such a deformation is a simple shear. In general, however, only special solutions ${ }^{1} u$ of (3.4) will be such that there exists a corresponding choice of $q$ (and thus of the hydrostatic pressure $p$ of (2.21)) satisfying (3.3). A material characterized by the strain energy density $W$ will be said to admit nontrivial states of anti-plane shear for a given pre-stretch $\lambda$ if, for every domain $\mathscr{D}$ and for every solution $u$ of (3.4), there is a function $q$ such that (3.3) holds ${ }^{2}$ for the given value of $\lambda$. The objective of the present section is the determination of a necessary and sufficient condition to be satisfied by $W$ if the associated material is to have this property.

The principal tool to be used in the proof of the theorem below is the hodograph transformation, in which

$$
\xi_{\alpha}=u, \alpha\left(x_{1}, x_{2}\right)
$$

become the new independent variables ${ }^{3}$. This transformation is assumed to define a one-to-one, smooth mapping from $\mathscr{D}$ in the physical plane to a domain $\Delta$ in the hodograph plane, in which $\xi_{\alpha}$ are rectangular cartesian coordinates. The associated Jacobian is

$$
H=u, 11 u, 22-u_{12}^{2}
$$

under the assumptions above, $H \neq 0$ on $\mathscr{D}$. It is convenient to introduce as a new unknown function the Legendre transform $U$ of $u$ by setting

$$
U\left(\xi_{1}, \xi_{2}\right)=x_{\beta} u,,_{\beta}\left(x_{1}, x_{2}\right)-u\left(x_{1}, x_{2}\right)=x_{\beta} \xi_{\beta}-u\left(x_{1}, x_{2}\right) .
$$

By differentiating (3.10) with respect to $\xi_{\alpha}$ and using (3.8), one obtains the relations

1. See the discussion on p. 85 of [8].

2. It is assumed that $u$ and $q$ are twice continuously differentiable on $\mathscr{D}$.

3. See $\$ 3$ of Chapter 1 of [6]. 


$$
\begin{gathered}
x_{\alpha}=\frac{\partial U}{\partial \xi_{\alpha}}\left(\xi_{1}, \xi_{2}\right) \\
u\left(x_{1}, x_{2}\right)=\xi_{\beta} \frac{\partial U}{\partial \xi_{\beta}}\left(\xi_{1}, \xi_{2}\right)-U\left(\xi_{1}, \xi_{2}\right)=\xi_{\beta} x_{\beta}-U\left(\xi_{1}, \xi_{2}\right),
\end{gathered}
$$

which are dual to (3.8), (3.10). If (3.11) is differentiated with respect to $x_{\beta}$, the resulting four simultaneous equations for $\partial \xi_{\gamma} / \partial x_{\beta}$ may be inverted to give the gradients of the mapping (3.8) in the form

$$
\frac{\partial \xi_{\alpha}}{\partial x_{\beta}}=u_{\alpha \beta}=H \varepsilon_{\alpha \lambda} \varepsilon_{\beta \mu} \frac{\partial^{2} U}{\partial \xi_{\lambda} \partial \xi_{\mu}} .
$$

The system (3.3), (3.4) for $u, q$ may now be converted with the help of (3.12), (3.13) to a set of three differential equations for $U$ and $Q$, where

$$
Q\left(\xi_{1}, \xi_{2}\right)=q\left(x_{1}, x_{2}\right) .
$$

One finds these equations to be

$$
\begin{gathered}
\left\{\frac{\partial Q}{\partial \xi_{\gamma}} \delta_{\alpha \beta}+\frac{\partial}{\delta \xi_{\gamma}}\left[G\left(R^{2} ; \lambda\right) \xi_{\alpha} \xi_{\beta}\right]\right\} \varepsilon_{\gamma \lambda} \varepsilon_{\beta \mu} \frac{\partial^{2} U}{\partial \xi_{\lambda} \partial \xi_{\mu}}=0 \text { on } \Delta, \\
\frac{\partial}{\partial \xi_{\gamma}}\left[F\left(R^{2} ; \lambda\right) \xi_{\beta}\right] \varepsilon_{\gamma \lambda} \varepsilon_{\beta \mu} \frac{\partial^{2} U}{\partial \xi_{\lambda} \partial \xi_{\mu}}=0 \text { on } \Delta,
\end{gathered}
$$

where

$$
R=|\nabla u|=\left(\xi_{\beta} \xi_{\beta}\right)^{\frac{1}{2}}
$$

If one introduces polar coordinates $R, \varphi$ in the hodograph plane by writing

$$
\xi_{1}=R \cos \varphi, \quad \xi_{2}=R \sin \varphi,
$$

one can transform the system (3.15), (3.16) to polar coordinates with the following result: (3.15) gives rise to

$$
\begin{gathered}
G R\left[R \frac{\partial}{\partial R}\left(\frac{1}{R} \frac{\partial U}{\partial R}\right)+\frac{2}{R^{2}} \frac{\partial^{2} U}{\partial \varphi^{2}}+\frac{3}{R} \frac{\partial U}{\partial R}\right]+2 G^{\prime}\left(R \frac{\partial^{2} U}{\partial \varphi^{2}}+R^{2} \frac{\partial U}{\partial R}\right) \\
+\frac{\partial Q}{\partial R}\left(\frac{1}{R^{2}} \frac{\partial^{2} U}{\partial \varphi^{2}}+\frac{1}{R} \frac{\partial U}{\partial R}\right)-\frac{1}{R} \frac{\partial Q}{\partial \varphi}\left(\frac{1}{R} \frac{\partial^{2} U}{\partial R \partial \varphi}-\frac{1}{R^{2}} \frac{\partial U}{\partial \varphi}\right)=0, \\
G R\left(\frac{1}{R} \frac{\partial^{2} U}{\partial R \partial \varphi}-\frac{1}{R^{2}} \frac{\partial U}{\partial \varphi}\right)+\frac{\partial Q}{\partial R}\left(\frac{1}{R} \frac{\partial^{2} U}{\partial R \partial \varphi}-\frac{1}{R^{2}} \frac{\partial U}{\partial \varphi}\right) \\
-\frac{1}{R} \frac{\partial Q}{\partial \varphi}\left[R \frac{\partial}{\partial R}\left(\frac{1}{R} \frac{\partial U}{\partial R}\right)+\frac{1}{R} \frac{\partial U}{\partial R}\right]=0,
\end{gathered}
$$

while the remaining differential equation (3.16) becomes 


$$
\frac{F}{F+2 R^{2} F^{\prime}} \frac{\partial^{2} U}{\partial R^{2}}+\frac{1}{R} \frac{\partial U}{\partial R}+\frac{1}{R^{2}} \frac{\partial^{2} U}{\partial \varphi^{2}}=0 .
$$

In (3.19)-(3.21), $G^{\prime}=G^{\prime}\left(R^{2} ; \lambda\right), F^{\prime}=F^{\prime}\left(R^{2} ; \lambda\right)$ stand for the derivatives of $F$ and $G$ with respect to $R^{2}$. It may be noted that the coefficient $F\left(F+2 R^{2} F^{\prime}\right)^{-1}$ in (3.21) is finite and positive for $R \geqq 0$ because of (3.5) and (3.6).

THEOREM. If the strain energy density $W\left(I_{1}, I_{2}\right)$ is such that (3.5) holds, then the associated incompressible elastic material admits nontrivial states of anti-plane shear for a given pre-stretch $\lambda$ if and only if $W$ also satisfies

$$
b \frac{\partial W}{\partial I_{1}}\left(I_{1}, I_{2}\right)+\left(\frac{b}{\lambda}-1\right) \frac{\partial W}{\partial I_{2}}\left(I_{1}, I_{2}\right)=0,
$$

for some constant $b$ which may depend on $\lambda$, for all values of $I_{1}, I_{2}$ such that

$$
I_{1}=\lambda^{2}+2 \lambda^{-1}+R^{2}, \quad I_{2}=\lambda^{-2}+2 \lambda+\lambda^{-1} R^{2}, \quad R \geqq 0 .
$$

Proof. To prove the necessity of (3.22), one first assumes that the material admits nontrivial states of anti-plane shear, constructs a solution of (3.4) for a special domain $\mathscr{D}$, and deduces (3.22) as necessary for the existence of a function $q$ satisfying (3.3). To carry out this task, it is useful to start with the linear equation (3.21) which is the hodograph version, in polar coordinates, of (3.4). It is easy to verify that

$$
U(R, \varphi)=V(R) \cos \varphi
$$

with

$$
V(R)=R \int_{R}^{\infty} \frac{d \rho}{\rho^{3} F\left(\rho^{2} ; \lambda\right)},
$$

is an exact solution ${ }^{2}$ of (3.21) on the domain $\Delta$ described by $R>0,0<\varphi<\pi$. The convergence of the integral in (3.25) is assured by the fact that, according to $(3.5), \rho F\left(\rho^{2} ; \lambda\right)$ is monotone increasing.

The solution $u$ of (3.4) which corresponds to (3.24) is obtained from (3.11), (3.12). If $r, \theta$ are polar coordinates in the physical plane, so that

$$
x_{1}=r \cos \theta, \quad x_{2}=r \sin \theta
$$

(3.11), (3.24) yield

1. Note that (3.22) need not hold for all values $I_{1} \geqq 3, I_{2} \geqq 3$, but merely for those values determined by (3.23) as $R$ varies from 0 to $\infty$ with $\lambda$ fixed at its given value. If, for example, there is no pre-stretch, so that $\lambda=1$, then (3.22) must hold only for those values of $I_{1}, I_{2}$ such that $I_{1}=I_{2} \geqq 3$.

2. Solutions of (3.21) which are independent of $\varphi$ are not useful for present purposes; they lead to axially symmetric solutions of (3.4) for which, as observed by Adkins [3], there always exists a function $q$ satisfying (3.3), regardless of the form of $W$. 


$$
\left.\begin{array}{l}
r \cos \theta=V^{\prime}(R) \cos ^{2} \varphi+\frac{1}{R} V(R) \sin ^{2} \varphi \\
r \sin \theta=\left[V^{\prime}(R)-\frac{1}{R} V(R)\right] \sin \varphi \cos \varphi
\end{array}\right\} \text { on } \Delta
$$

while (3.12), (3.24) furnish

$$
u=\left[R V^{\prime}(R)-V(R)\right] \cos \varphi
$$

It is possible to show by calculations too lengthy to include here that (3.27) provides a one-to-one mapping from the half-plane $\Delta: R>0,0<\varphi<\pi$, onto the domain $\mathscr{D}$ in the physical plane defined by

$$
\mathscr{D}: r>0,-\pi<\theta<\pi
$$

Thus $\mathscr{D}$ consists of the entire $x_{1}, x_{2}$ plane cut along the negative real axis. ${ }^{1}$ In establishing the one-to-one nature of the transformation (3.27), one makes essential use of the ellipticity condition (3.5). Equations (3.26), (3.27), (3.28) then implicitly furnish a solution $u$ of the original differential equation (3.4) on the domain $\mathscr{D}$ of (3.29).

By assumption, there exists a function $q$ on $\mathscr{D}$ satisfying (3.3) when $u$ is chosen to be the solution of (3.4) constructed above. If $Q$ is the hodograph image of $q$ (see (3.14)), the one-to-one nature of the mapping $\mathscr{D} \leftrightarrow \Delta$ assures that $Q$ must satisfy (3.19) and (3.20) on $\Delta$ when $U$ is given by (3.24), (3.25). Substitution from (3.24) into (3.19), (3.20) yields, after some manipulation, the equations

$$
\left.\begin{array}{r}
{\left[F \frac{\partial \hat{Q}}{\partial R}+2 R^{3}\left(F G^{\prime}-G F^{\prime}\right)\right] \cos \varphi+\frac{F}{R} \frac{\partial \hat{Q}}{\partial \varphi} \sin \varphi=0} \\
F \frac{\partial \hat{Q}}{\partial R} \sin \varphi-\left(F+2 R^{2} F^{\prime}\right) \frac{1}{R} \frac{\partial \hat{Q}}{\partial \varphi} \cos \varphi=0
\end{array}\right\}
$$

where the abbreviation

$$
\hat{Q}=Q+\int_{0}^{R} G\left(\rho^{2} ; \lambda\right) \rho d \rho
$$

has been introduced. Solving (3.30) for the derivatives of $\hat{Q}$, one obtains

1. As suggested by the form of the domain $\mathscr{D}$, the solution (3.24) plays a major role in the treatment of a crack problem in finite anti-plane shear; see $\$ 6$ of [10]. 


$$
\left.\begin{array}{l}
\frac{\partial \hat{Q}}{\partial R}=\frac{-f g \cos ^{2} \varphi}{f \cos ^{2} \varphi+\sin ^{2} \varphi}, \\
\frac{\partial \hat{Q}}{\partial \varphi}=\frac{-R g \sin \varphi \cos \varphi}{f \cos ^{2} \varphi+\sin ^{2} \varphi},
\end{array}\right\}
$$

where

$$
\begin{aligned}
& f=f(R ; \lambda)=1+\frac{2 R^{2} F^{\prime}\left(R^{2} ; \lambda\right)}{F\left(R^{2} ; \lambda\right)}>0 \text { for } \quad R \geqq 0 . \\
& g=g(R ; \lambda)=\frac{2 R^{3}\left[F\left(R^{2} ; \lambda\right) G^{\prime}\left(R^{2} ; \lambda\right)-G\left(R^{2} ; \lambda\right) F^{\prime}\left(R^{2} ; \lambda\right)\right]}{F\left(R^{2} ; \lambda\right)}, \quad R \geqq 0 .
\end{aligned}
$$

Elimination of $\hat{Q}$ from (3.32) by cross-differentiation gives

$$
\left[R\left(f g^{\prime}-g f^{\prime}\right)+3 f g\right] \cos ^{2} \varphi+\left(R g^{\prime}+2 f g+g\right) \sin ^{2} \varphi=0, \quad R \geqq 0
$$

It follows that $f$ and $g$ must satisfy the two ordinary differential equations

$$
\begin{gathered}
R\left(f g^{\prime}-g f^{\prime}\right)+3 f g=0, \quad R \geqq 0, \\
R g^{\prime}+g+2 f g=0, \quad R \geqq 0 .
\end{gathered}
$$

Let

$$
h(R ; \lambda)=\frac{g(R ; \lambda)}{f(R ; \lambda)}, \quad R \geqq 0,
$$

note from (3.33), (3.34) that

$$
h(0 ; \lambda)=0,
$$

and observe that (3.36) can be written as

$$
R h^{\prime}+3 h=0, \quad R \geqq 0 .
$$

The initial value problem (3.40), (3.39) has the unique solution $h \equiv 0$; from (3.38) one then concludes that

$$
g \equiv 0, \quad R \geqq 0, \quad \lambda>0 .
$$

Reference to (3.34) shows that (3.41) implies

$$
F G^{\prime}-F^{\prime} G=0, \quad R \geqq 0, \quad \lambda>0,
$$

so that

1. It may be noted that (3.37), as well as (3.36), is satisfied when $g \equiv 0$. 


$$
G=b F, \quad R \geqq 0, \quad \lambda>0,
$$

where $b$ is a constant which may depend on $\lambda$. From (3.43) and the definitions (3.1) of $F$ and $G$, one concludes that

$$
\frac{\partial W}{\partial I_{2}}\left(I_{1}, I_{2}\right)=b\left[\frac{\partial W}{\partial I_{1}}\left(I_{1}, I_{2}\right)+\frac{1}{\lambda} \frac{\partial W}{\partial I_{2}}\left(I_{1}, I_{2}\right)\right],
$$

provided

$$
I_{1}=\lambda^{2}+2 \lambda^{-1}+R^{2}, \quad I_{2}=\lambda^{-2}+2 \lambda+\lambda^{-1} R^{2} .
$$

The necessity of (3.22), (3.23) is therefore established.

To show that (3.22), (3.23) provide a sufficient condition for the existence of nontrivial states of anti-plane shear, one observes that, if these conditions hold, then so does (3.43), so that (3.3) may now be written as

$$
q_{,_{\alpha}}+b\left(F u,_{\alpha} u,_{\beta}\right)_{,_{\beta}}=0 \text { on } \mathscr{D} \text {. }
$$

Now suppose that $\mathscr{D}$ is an arbitrary domain and $u$ an arbitrary twice continuously differentiable solution of $(3.4)$ on $\mathscr{D}$. Then

$$
q,_{\alpha}+b\left(F u,_{\alpha} u,_{\beta}\right)_{,_{\beta}}=q_{,_{\alpha}}+b F u,_{\beta} u_{, \beta \alpha}=q_{, \alpha}+\frac{b}{2}\left(|\nabla u|^{2}\right)_{, \alpha} F \text { on } \mathscr{D} .
$$

Define

$$
q=-\frac{b}{2} \int_{0}^{R^{2}} F(\rho ; \lambda) d \rho, \quad R \geqq 0
$$

where

$$
R=|\nabla u|
$$

Then, by (3.47), $q$ and $u$ satisfy (3.46) on $\mathscr{D}$, so that the material characterized by the strain energy density $W$ does indeed admit nontrivial states of anti-plane shear.

This completes the proof of the theorem.

\section{Discussion}

A number of observations may be made immediately concerning the condition (3.22), (3.23) restricting the form of $W$.

(i) Examples Materials with a strain energy density of the form

$$
W=\hat{W}\left(I_{1}\right)
$$

1. An arbitrary constant can be added to $q$. 
clearly satisfy (3.22), (3.23) for every $\lambda>0$ with $b=0$. The Mooney material described by (2.26) also satisfies (3.22), (3.23) for every $\lambda>0$, provided $A>0$, $B \geqq 0$; in this case one has

$$
b=B\left(A+\frac{1}{\lambda} B\right)^{-1} .
$$

Thus both of these types of materials admit nontrivial states of anti-plane shear for all values of the axial pre-stretch $\lambda$.

The most general material which has this latter property and for which $b$ is given by (4.2) is easily shown to be characterized by a strain energy density of the form

$$
W=\hat{W}\left(A I_{1}+B I_{2}\right)
$$

where $A>0$ and $B \geqq 0$ are constants, and $\hat{W}$ is an arbitrary sufficiently smooth function. Materials for which $W$ has the form (4.3) thus admit nontrivial states of anti-plane shear for every $\lambda>0$ and at the same time generalize both the Mooney material and materials characterized by (4.1).

If $W$ is of the form (2.28), it is easy to show that (3.22) and (3.23) hold if $\lambda=1$, but they are not in general satisfied when $\lambda \neq 1$. Thus, as pointed out by Lo [13], materials associated with a strain energy density of the form (2.28) admit nontrivial states of anti-plane shear, but only in the absence of axial pre-stretch.

An example of a material for which nontrivial states of anti-plane shear are not permitted for any value of the axial pre-stretch is provided by strain energy densities of the form

$$
W=A I_{1}+\hat{W}\left(I_{2}\right)
$$

in which $\boldsymbol{A}$ is a constant and $\hat{W}$ is a function whose second derivative does not vanish identically for $I_{2} \geqq 3$. One shows readily in this instance that (3.22), (3.23) cannot hold for any $\lambda>0$, no matter how $b$ may be chosen.

(ii) The pressure gradient case Although the considerations leading to the necessity and sufficiency of (3.22), (3.23) were based on the assumption of vanishing axial pressure gradient $(c=0)$ in the equilibrium equation (2.24), it is nevertheless possible to verify that, if (3.22), (3.23) hold for a particular $\lambda$, then, for any solution $u$ of $(2.24)$, the choice

$$
q=c\left(1-\lambda^{-1} b\right) u-\frac{1}{2} \int_{0}^{R^{2}} F(\rho ; \lambda) d \rho, \quad R=|\nabla u|
$$

1. Compare with (3.48). 
always assures that (2.23) is satisfied for that $\lambda$, even if $c \neq 0$. Here $F$ is as defined in (3.1). Thus (3.22), (3.23) remain sufficient for the existence of nontrivial states of anti-plane shear in the presence of an axial pressure gradient; they might, however, not be necessary.

(iii) Stresses Suppose that a material with strain energy density $W\left(I_{1}, I_{2}\right)$ admits nontrivial states of anti-plane shear for a fixed $\lambda>0$, so that (3.22), (3.23) hold. From (3.48) and (2.21) it follows that the hydrostatic pressure $p$ is given (for $c=0$ ) by

$$
\left.\begin{array}{c}
p=-\frac{b}{2 \lambda} \int_{0}^{R^{2}} F(\rho ; \lambda) d \rho+\frac{1}{\lambda}\left[1+b\left(\lambda^{2}+R^{2}\right)\right] F\left(R^{2} ; \lambda\right), \\
R=|\nabla u| .
\end{array}\right\}
$$

One may now determine from (4.6), (2.15)-(2.18), and (2.7), (2.10) the expressions for the nominal and true stresses $\sigma_{i \jmath}$ and $\tau_{t,}$, respectively. Making use of (3.22) as necessary, one finds for $\sigma_{i j}$

$$
\begin{gathered}
\sigma_{\alpha \beta}=\lambda^{-\frac{1}{2}} \frac{b}{2} \int_{0}^{R^{2}} F(\rho ; \lambda) d \rho \delta_{\alpha \beta}-\lambda^{-\frac{1}{2}} b F\left(R^{2} ; \lambda\right) u, u_{\alpha, \beta}, \\
\sigma_{\alpha^{3}}=\left\{-\lambda^{-\frac{1}{2}} \frac{b}{2} \int_{0}^{R^{2}} F(\rho ; \lambda) d \rho+\lambda^{-\frac{3}{2}}\left(1+b R^{2}\right) F\left(R^{2} ; \lambda\right)\right\} u_{, \alpha}, \\
\sigma_{3 \alpha}=F\left(R^{2} ; \lambda\right) u_{, \alpha}, \\
\sigma_{33}=\lambda^{-2} \frac{b}{2} \int_{0}^{R^{2}} F(\rho ; \lambda) d \rho+\left(\lambda-\lambda^{-2}-\lambda^{-2} b R^{2}\right) F\left(R^{2} ; \lambda\right),
\end{gathered}
$$

where $F$ is defined in (3.1) and $R$ is given by the second of (4.6). For the true stresses, one has

$$
\begin{gathered}
\tau_{\alpha \beta}=\lambda^{-\frac{1}{2}} \sigma_{\alpha \beta}, \\
\tau_{\alpha 3}=\tau_{3 \alpha}=\lambda^{-\frac{1}{2}} \sigma_{3 \alpha}, \\
\tau_{33}=-\lambda^{-2} \frac{b}{2} \int_{0}^{R^{2}} F(\rho ; \lambda) d \rho+\left[\left(1-\lambda^{-1} b\right) R^{2}+\lambda^{2}-\lambda^{-1}\right] F\left(R^{2} ; \lambda\right) .
\end{gathered}
$$

When $b=0$ (so that (4.1) holds) and $\lambda=1,(4.7)-(4.13)$ reduce to corresponding results in $[10]^{2}$. If the material is of Mooney type, so that $W$ is given by (2.26), the true stresses (4.11)-(4.13), when expressed explicitly in terms of $u_{, a}$ with the aid of (4.7)-(4.10), coincide with those given in [8] ${ }^{3}$.

1. An arbitrary constant may, of course, be added to $p$.

2. See Eq. (3.3) of $[10]$.

3. See Eq. (2.20.18) of [8]; allowance must be made for differences in notation. 


\section{Acknowledgement}

The results communicated in this paper were obtained in the course of an investigation supported under Contract N00014-75-C-0196 between the California Institute of Technology and the Office of Naval Research.

\section{References}

[1] J. D. Achenbach, 'Bifurcation of a running crack in anti-plane strain', International Journal of Solids and Structures, 11 (1975), 1301.

[2] J. D. Achenbach and Z. P. Bazant, "Elastodynamic near-tip stress and displacement fields for rapidly propagating cracks in orthotropic materials', J. Appl. Mech. 42 (1975), 252.

[3] J. E. Adkins, 'Some generalizations of the shear problem for isotropıc incompressible materials', Proceedings of the Cambridge Philosophical Society, 50 (1954), 334.

[4] J. C. Amazigo, 'Fully plastic crack in an infintte body under anti-plane shear', International Journal of Solids and Structures, 10 (1974), 1003.

[5] J. C. Amazigo, 'Fully plastic center-cracked strip under anti-plane shear', International Journal of Solids and Structures, 11 (1975), 1291.

[6] L. Bers, Mathematical aspects of subsonic and transonic gas dynamics, Surveys in Applied Mathematics, Vol. 3, Wiley, New York, 1958.

[7] R. Courant and D. Hilbert, Methods of Mathematical Physics, Vol. II, Interscience, New York, 1962.

[8] A. E. Green and J. E. Adkins, Large Elastic Deformatıons, Clarendon Press, Oxford, 1960.

[9] J. A. H. Hult and F. A. McClintock, 'Elastıc-plastic stress and strain distribution around sharp notches under repeated shear', Proceedings of the Ninth International Congress of Applied Mechanics, Brussels, 8 (1956), 51.

[10] J. K. Knowles, 'The finite anti-plane shear field near the tip of a crack for a class of incompressible elastıc solids' to appear in International Journal of Fracture, 13 (1977).

[11] J. K. Knowles and E Sternberg, 'An asymptotic finite-deformation analysis of the elastostatic field near the tip of a crack', Journal of Elasticity, 3 (1973), 67.

[12] J. K. Knowles and E. Sternberg, 'Finite-deformation analysis of the elastostatic field near the tip of a crack: Reconsideration and higher order results', Journal of Elasticity, 4 (1974), 201.

[13] K. K Lo, 'Finite deformation crack in an infinite body under anti-plane simple shear', to appear in International Journal of Solids and Structures.

[14] J. R Rice, 'Stresses due to a sharp notch in a work-hardening elastic-plastıc material loaded by longitudinal shear', J. Appl. Mech., 34 (1967) 287.

[15] C. Truesdell and W. Noll, 'The nonlinear field theories of mechanics', Handbuch der Physik, Vol. III/1, Springer, Berlin, 1965.

Division of Engineering and Applied Science,

California Institute of Technology,

Pasadena, California,

U.S.A. 